\title{
Modeling of Sandia Piloted Flame D and F using Flamelet-Generated Manifolds
}

\author{
Jun Tang a, Wenyan Song ${ }^{b}$ \\ School of Power and Energy, Northwestern Polytechnical University, Xi'an 710072, China \\ atangjun207@163.com, bwenyan_song@nwpu.edu.cn
}

\begin{abstract}
Keywords: Flamelet Generated Manifolds, Cantera, $\beta-P D F$, Piecewise Integration Method, extinction and re-ignition
\end{abstract}

\begin{abstract}
For purpose of studying the application of Flamelet Generated Manifolds (FGM) model in numerical simulation of combustion, the combustion field of Sandia piloted flame D and F were studied using FGM model. The laminar flamelet database was generated using the laminar 1D freely propagating flame module of open source Cantera, and the chemical kinetic mechanism used GRI-Mech 2.11. The interaction of turbulence and combustion was taken into account utilizing $\beta-\operatorname{PDF}($ Probability Density Function), the integration of which was treated with Piecewise Integration Method(PIM), and the turbulent characteristics was captured with Realizable $\kappa-\varepsilon$ model. The comparison between the numerical method and experiments shows: FGM model can model the mixing and combustion characteristics of Flame $\mathrm{D}$ and $\mathrm{F}$ including prominent extinction and re-ignition phenomenon with high accuracy.
\end{abstract}

\section{Introduction}

The requirement of higher performance of combustion and stricter regulation about emissions promotes the application of the numerical method in the combustor design. The accurate simulation of combustion field quite contributes to the optimization and design of combustion equipment.

In order to acquiring the effective information of combustion field, it is necessary to take detailed chemical mechanism into account, and this however is a very large computation cost in current CFD model, and almost is infeasible especially in practical combustion equipments. Therefore it is essential to reduce the model of chemical reaction model, and at present there is two kinds of simplified method: one is separation of fast and slow reactions, such ILDM (Intrinsic Low-Dimensional Manifolds) [1] method; the other one is based on flamelet method, such SLFM (Steady Laminar Flamelet Model) [2] and FPV (Flamelet/Progress Variable) [3-4]. The first method usually involves the influence of chemical kinetics and ignores the effect of diffusion resulting in lower accuracy in low temperature. The models based on flamelet method achieve great success in simplified chemical kinetics modeling. FGM [5-6] model combining the advantages of the two methods is a combustion modeling methods of great application prospects.

This paper reports a numerical study of the combustion field of Sandia piloted flame D and F using FGM model combined with $\beta$-PDF [7], and the GRI-Mech 2.11[8] is used as the chemical mechanism. The turbulent feature is captured through Realizable $\kappa-\varepsilon$ model.

\section{Mathematical Model}

Realizable $\kappa-\varepsilon$ model is used as the turbulent modeling, which can guarantee the realizability of normal stress compared with Standard $\kappa-\varepsilon$, and is an effective two-equations turbulent model. The detailed process references Shih [9].

Flamelet Generated Manifolds solves the laminar 1D freely propagating premixed flame at different mixing state which denoted by mixture fraction based on the concept of flamelet, and then the thermo-chemical parameters $\Phi$, such as species mass fraction, chemical reaction source, temperature 
and density, are mapped into two control variables, namely mixture fraction $\mathrm{Z}$ and progress variable $\mathrm{C}$, as shown in equation (1).

$$
\Phi=\Phi(Z, C)
$$

So the laminar flamelet database is formed. In general the progress variable $\mathrm{C}$ is the combination of species mass fraction, in current paper, $\mathrm{C}$ is defined as $C=y / y_{\text {eq }}$ in which $\mathrm{y}$ is sum of the mass fraction of $\mathrm{CO}_{2}, \mathrm{H}_{2} \mathrm{O}, \mathrm{CO}$ and $\mathrm{H}_{2}$, and the subscript 'eq' stands for the equilibrium state.

The laminar flamelet database is generated through the laminar 1D freely propagating flame module of the open source kinetics and flame simulation code Cantera [10], and GRI-Mech 2.11 is used as the chemical mechanism. In turbulent flame, the mean flamelet parameters are the integration of the thermo-chemical parameters in laminar flamelet database and join PDF of mixture fraction and progress variable

$$
\tilde{\Phi}=\iint \Phi(Z, C) P(Z, C) d Z d C
$$

Where the PDFs of $Z$ and $C$ are assumed statically independent. $\beta$-PDF is used as the PDF of mixture fraction and $\delta$-PDF is for progress variable, so

$$
\tilde{\Phi}=\int P(C) d C \int \Phi(Z, C) P(Z) d Z
$$

Because of inclusion of mean mixture fraction $\tilde{Z}$ and mixture fraction $\tilde{Z}^{\prime 2}$, so the turbulent flamelet database after integration is $\tilde{\Phi}=\tilde{\Phi}\left(\tilde{Z}, \tilde{Z}^{\prime 2}, \tilde{y}\right)$. In the turbulent flame only the transport equations for $\tilde{Z}$, $\tilde{Z}^{\prime 2}$ and $\tilde{y}$ are solved, and then the turbulent thermo-chemical parameters are attained through tri-linear interpolation from turbulent flamelet database.

The integration of equation (3) is quite difficult because of the characteristic of $\beta$-PDF. In current paper, Piecewise Integration Method which is an effective, steady and accuracy algorithm, is used as the integration method of $\beta$-PDF.

The transport equations of $\tilde{Z}, \tilde{Z}^{\prime \prime 2}$ and $\tilde{y}$ as shown in equation (4) (6), respectively.

$$
\begin{aligned}
& \frac{\partial\left(\bar{\rho} \tilde{u}_{i} \tilde{Z}\right)}{\partial x_{i}}=\frac{\partial}{\partial x_{i}}\left[\left(\bar{\rho} D_{Z}+\frac{\mu_{t}}{\sigma_{z}}\right) \frac{\partial \tilde{Z}}{\partial x_{i}}\right] \\
& \frac{\partial\left(\bar{\rho} \tilde{u}_{i} \tilde{Z}^{\prime \prime 2}\right)}{\partial x_{i}}=\frac{\partial}{\partial x_{i}}\left[\left(\bar{\rho} D_{Z}+\frac{\mu_{t}}{\sigma_{z}}\right) \frac{\partial \tilde{Z}^{\prime \prime 2}}{\partial x_{i}}\right]+C_{g 1} \mu_{t}\left(\frac{\partial \tilde{Z}^{\prime \prime 2}}{\partial x_{i}}\right)^{2}-C_{g 2} \frac{\tilde{\varepsilon}}{\tilde{k}} \bar{\rho} \tilde{Z}^{\prime \prime 2} \\
& \frac{\partial\left(\bar{\rho} \tilde{u}_{i} \tilde{y}\right)}{\partial x_{i}}=\frac{\partial}{\partial x_{i}}\left[\left(\bar{\rho} D_{C}+\frac{\mu_{t}}{\sigma_{y}}\right) \frac{\partial \tilde{y}}{\partial x_{i}}\right]+\bar{\rho} \tilde{\dot{\omega}}_{y}
\end{aligned}
$$

Where $\mathrm{C}_{\mathrm{g} 1}=2.86, \mathrm{C}_{\mathrm{g} 2}=2.0$ and $\sigma_{\mathrm{z}}=\sigma_{\mathrm{y}}=0.7$.

\section{Experimental and Numerical Setup}

The Sandia piloted flame D and F are used for the simulation. The burner is located in co-flow of 0.9 $\mathrm{m} / \mathrm{s}$ velocity, as shown in Fig. 1 and the co-flow temperature is $294 \mathrm{~K}$. The fuel jet is comprised of methane and air with a volume ratio of $1: 3$ and temperature of $294 \mathrm{~K}$ and the diameter is $7.2 \mathrm{~mm}(\mathrm{~d})$. The piloted jet is comprised of a burned mixture of $\mathrm{C}_{2} \mathrm{H}_{2}, \mathrm{H}_{2}$, air, $\mathrm{CO}_{2}$ and $\mathrm{N}_{2}$ with temperature of $1880 \mathrm{~K}$ and the inner and outer diameter are 7.7 and 18.2 respectively. The ambient pressure is 1 bar. The velocity of fuel of flame $\mathrm{D}$ and $\mathrm{F}$ are $49.2 \mathrm{~m} / \mathrm{s}$ and $99.2 \mathrm{~m} / \mathrm{s}$ respectively, and the velocity of piloted jet is $11.4 \mathrm{~m} / \mathrm{s}$ and $22.8 \mathrm{~m} / \mathrm{s}$ respectively.

The computational domain is the sector of $30^{\circ}$ in which the axial direction is started from the exit of injector, and extended to $80 \mathrm{~d}$ downstream, and radial direction is extended to $20 \mathrm{~d}$ location, as shown in Fig. 2. There is dramatic extinction and re-ignition in flame F, as show from Fig. 3 and Fig. 4 

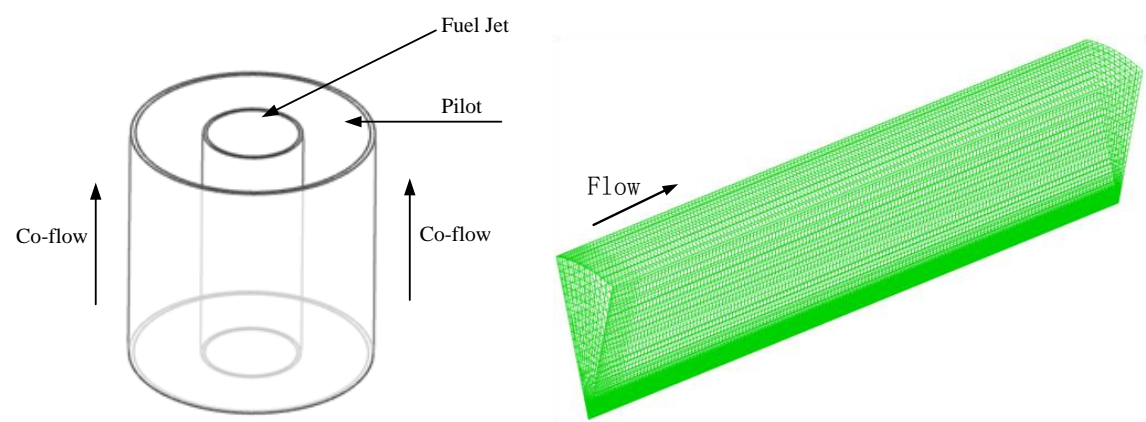

Fig. 1 Sandia piloted burner configuration Fig. 2 The mesh for numerical simulation

\section{Results and Discussions}

Figure 3 and 4 show the radial distribution of mean mixture fraction and temperature respectively at different axial location. As shown in the figures, the distribution of mean mixture fraction and temperature can be simulated by FGM model with high accuracy. At $\mathrm{x} / \mathrm{d}=2$, there is some deviation around $\mathrm{r} / \mathrm{d}=1$, and this is related to the inaccurate boundary condition of piloted jet. At $\mathrm{x} / \mathrm{d}=30$ which is alongside the end of fuel jet, because the penetration depth is overestimated by Realizable $\kappa-\varepsilon$ model, the mean mixture fraction around axis is overestimated and yet temperature is underestimated. There is extinction and re-ignition phenomenon in flame F, and FGM model can simulate the mixture and combustion characteristic of flame F, so FGM model can capture the extinction and re-ignition phenomenon.
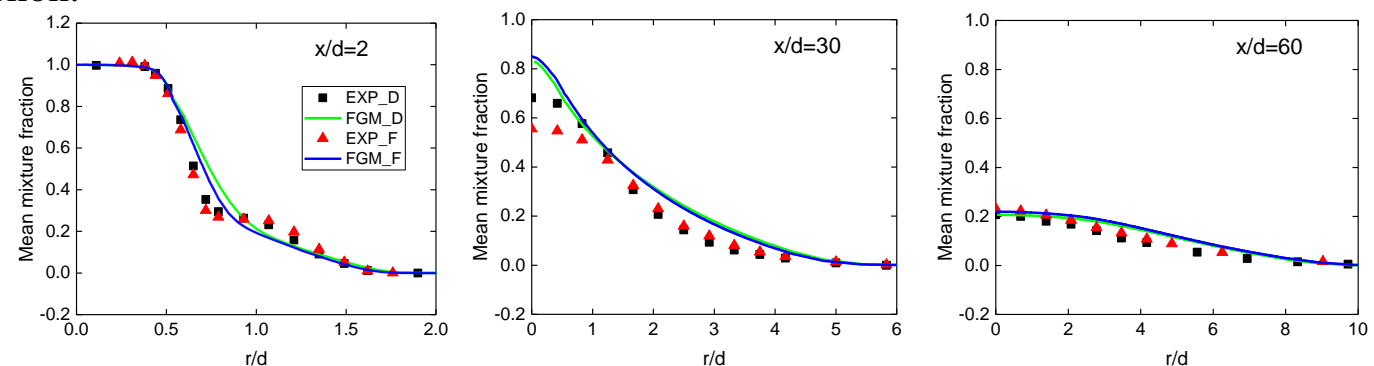

Fig. 3 Radial profile of mean mixture fraction at different axial location

Figure 5 and Figure 6 illustrate the radial distribution of $\mathrm{CO}_{2}$ and $\mathrm{H}_{2} \mathrm{O}$ mass fraction respectively at different axial location. As shown from the figures, the simulation has a good agreement with experiment results, indicating that FGM can model the distribution of species mass fraction with high accuracy. However the modeling deviation of mean mixture fraction and temperature also reflects the distribution of species mass fraction, and the influence on $\mathrm{CO}_{2}$ is especially obvious.
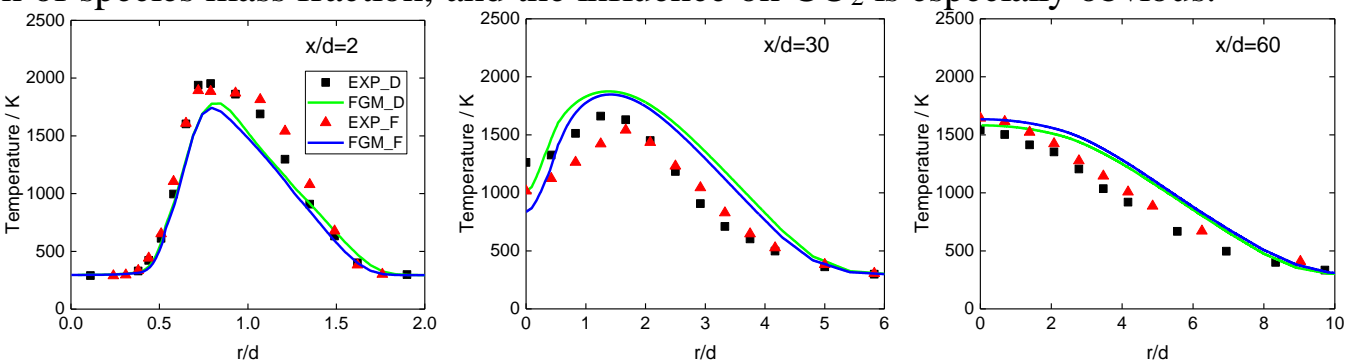

Fig. 4 Radial profile of temperature at different axial location
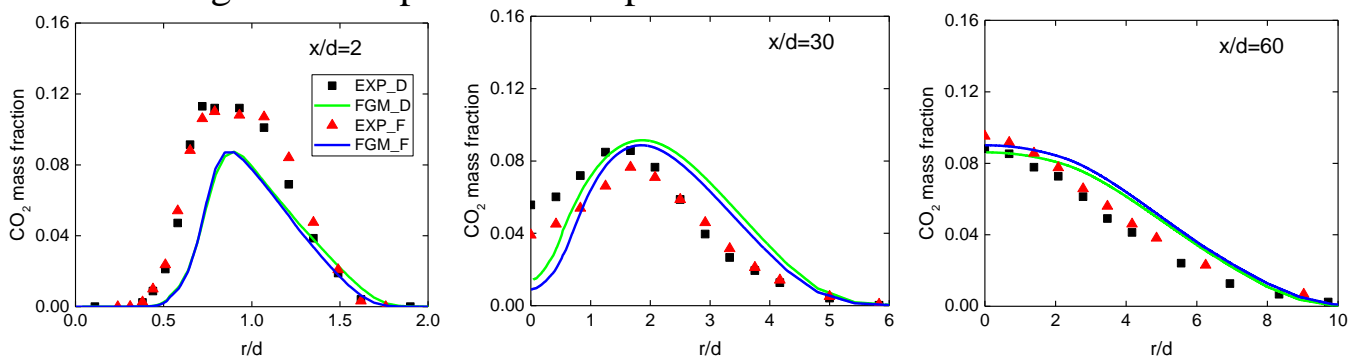

Fig. 5 Radial profile of $\mathrm{CO} 2$ mass fraction at different axial location 

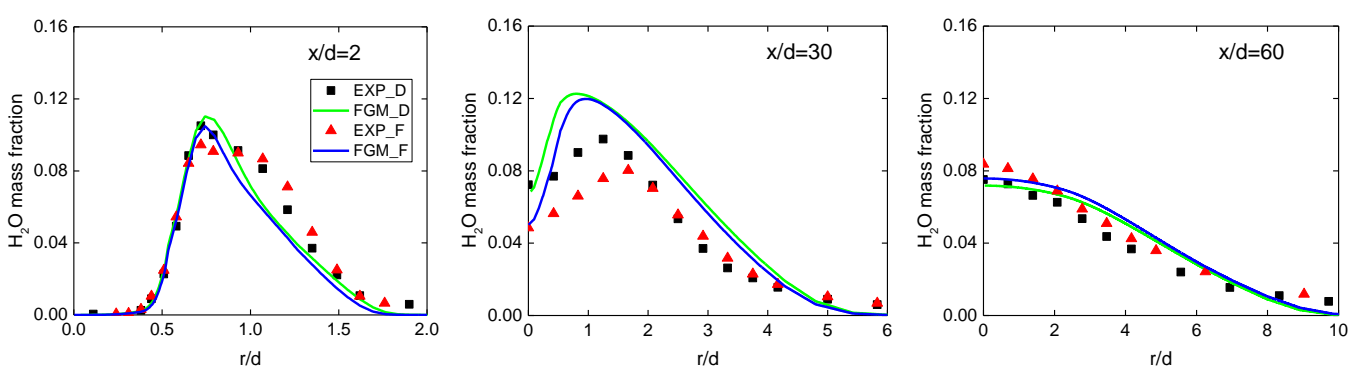

Fig. 6 Radial profile of $\mathrm{H} 2 \mathrm{O}$ mass fraction at different axial location

\section{Conclusion}

This paper reports the simulation of Sandia piloted flame D and F using FGM model, indicating:

(1) FGM can effectively model the mixing and combustion characteristic of the steady piloted flame

$\mathrm{D}$ and flame $\mathrm{F}$ with obvious extinction and re-ignition;

(2) FGM can model temperature and species mass fraction distribution with high accuracy.

\section{References}

[1]. Bongers H: Analysis of Flamelet-Based Methods to Reduce Chemical Kinetics in Flame Computations (Master, Technische Universiteit Eindhoven, Holland 2005). p.87.

[2]. Claramunt K, Consul R, Carbonell D, et al. Laminar Flamelet Concept for Laminar and Turbulent Diffusion Flames. AIAA-2004-796.Heyl A, Bockhorn. Flamelet Modeling of NO Formation in Laminar and Turbulent Diffusion Flames. Vol. 42 (2001) No. 5-7, 449-462.

[3]. Pierce C D: Progress-Variable Approach for Large-Eddy Simulation of Turbulent Combustion (Doctor, Stanford University, USA 2001). p.26.

[4]. Ravikanti M: Advanced Flamelet Modeling of Turbulent Nonpremixed and Partially Premixed Combustion (Doctor, Loughborough University, UK 2008). p.51.

[5]. Van Oijen J A: Flamelet-Generated Manifolds: Development and Application to Premixed Laminar Flames (Doctor, Technische Universiteit Eindhoven, Holland 2002). p.17.

[6]. Ramaekers W J S: Development of Flamelet Generated Manifolds for Partially-Premixed Flame (Doctor, Technische Universiteit Eindhoven, Holland 2011). p.13.

[7]. Lien F S, Liu H, Chui E, et al. Development of an Analytical $\beta$-Function PDF Integration Algorithm for Simulation of Non-Premixed Turbulent Combustion. Flow, Turbulence and Combustion. Vol. 83 (2009) No. 2, p. 205-226.

[8]. Information on: http://www.me.berkeley.edu/gri_mech

[9]. Shih T H, Liou W W, Shabbir A, et al. A New $\kappa-\varepsilon$ Eddy Viscosity Model for High Reynolds Number Turbulent Flows. Comput Fluids. Vol. 24 (1995) No. 3, 227-238.

[10]. Information on: http://www.cantera.org 